Supporting Information

\title{
Substantial Improvement of Oil Aerosol Filtration Performance using in-plane Asymmetric Wettability
}

Xin Weit, Yufeng Liut, Hua Zhout, Feng Chent, Hongxia Wang ${ }^{\star}$, Zhongli Jit, George G. Chases, Tong Lin ${ }^{\text {*t }}$

† Institute for Frontier Materials, Deakin University, Geelong, VIC 3216, Australia.

$\ddagger$ Beijing Key Laboratory of Process Fluid Filtration and Separation, College of Mechanical and Transportation Engineering, China University of Petroleum, Beijing 102249, PR China. $\S$ Department of Chemical and Biomolecular Engineering, the University of Akron, Akron, $\mathrm{OH}$ 44325, USA.

(Corresponding Authors' emails: hong.wang@deakin.edu.au; tong.lin@deakin.edu.au) 


\section{Estimation of the capillary radius $(\eta)$ :}

For porous materials, the rate of liquid penetration can be expressed as below based on Poiseuille's Law ${ }^{1-2}$ :

$$
\frac{\partial l}{\partial t}=\frac{r^{2}}{8 \eta l}\left(\frac{2 \gamma}{r} \cos \theta-\rho g l\right)
$$

In this equation, / is the distance of liquid penetrated the cylinder tube, which refers to the vertical wicking height in this work. The total pressure difference $\left(\frac{2 \gamma}{r} \cos \theta-\rho g l\right)$ is the difference between capillary pressure $\left(P_{c}=\frac{2 \gamma}{r} \cos \theta\right)$ and hydrostatic pressure $\left(P_{h}=\rho g l\right)$.

While the hydrostatic pressure is neglected in case of flow under capillary pressure. Equation (S1) is simplified as:

$$
l \frac{\partial l}{\partial t}=\frac{r \gamma \cos \theta}{4 \eta}
$$

The idealized equivalent capillary diameter $(D)$ is obtained in equation (S2), as shown in the manuscript.

\section{References}

1. Washburn, E. W., The Dynamics of Capillary Flow. Physical Review, 1921, 17 (3), 273283.

2. Dang $\mathrm{Vu}, \mathrm{T}$.; Jan, $\mathrm{H}$. , Characterization of porous materials by capillary rise method. Physicochem. Probl. Mi. 2005, 39. 

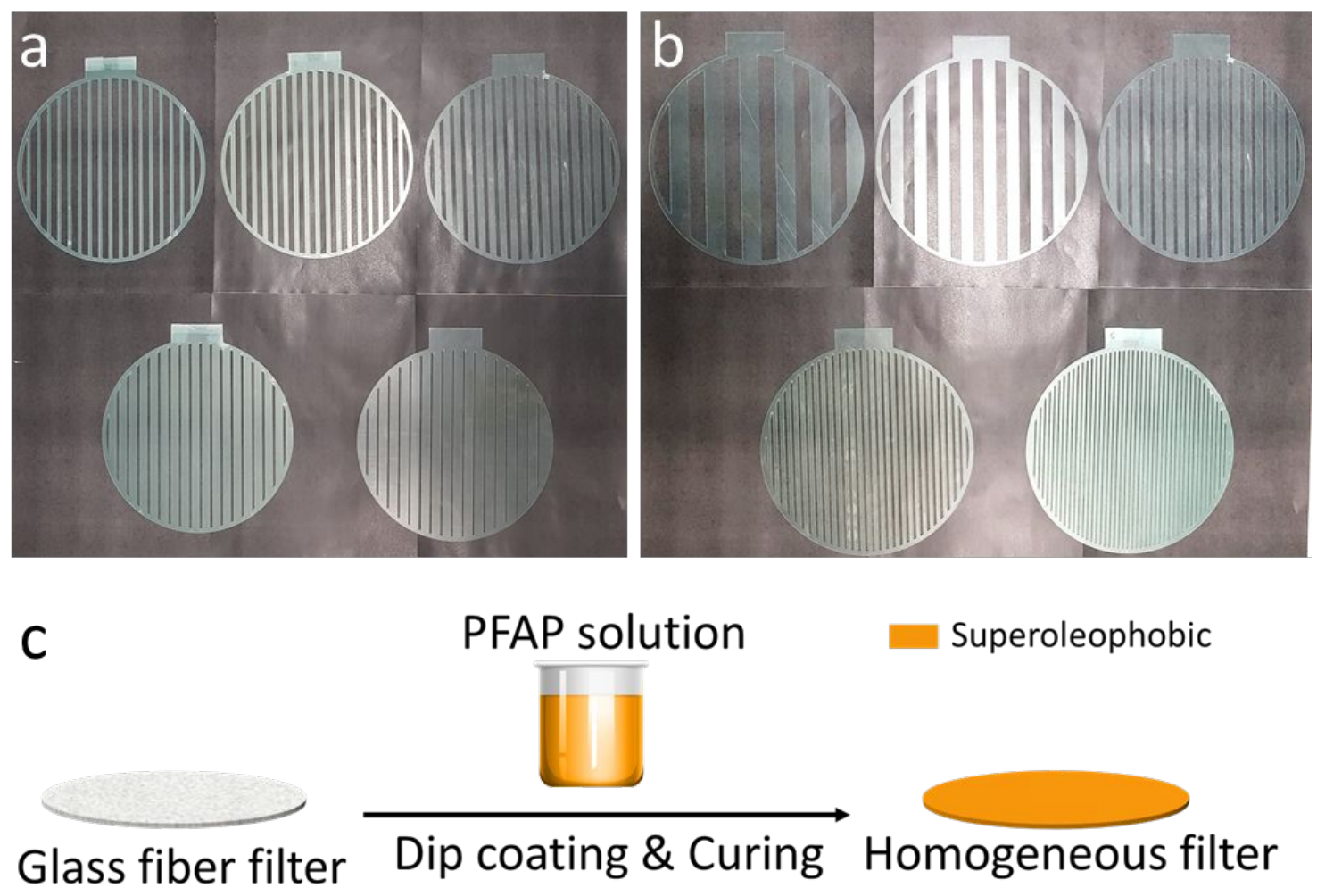

Figure S1. (a-b) Digital photos of plastic masks for spray coating (more details in Table S1) (a) masks for Series / (b) masks for Series II. (c) Preparation method of the homogeneous superoleophobic surface. 

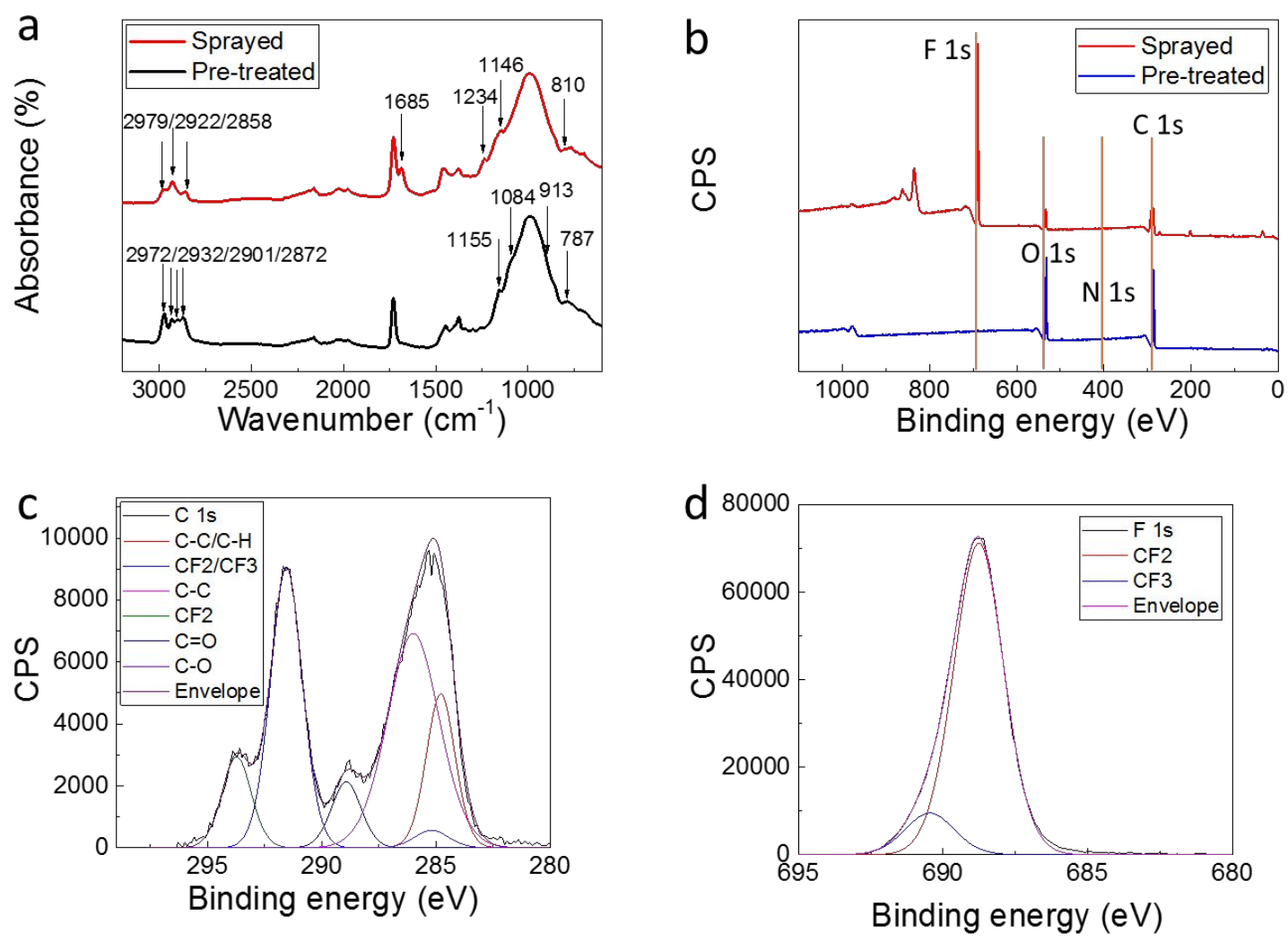

Figure S2. Chemical characterization of pre-treated and spray-coated glass fiber filter (a) FTIR spectrum (b) XPS survey spectra, (c) High-resolution C1s spectra of spray-coated glass fibers, (d) High-resolution F1s spectra of spray-coated glass fibers.

Assignment of FTIR spectra

\begin{tabular}{c|c}
\hline Wavenumber $\left(\mathrm{cm}^{-1}\right)$ & Vibrations \\
\hline 810 & Si-O in fluoroalkylsilane \\
913 and 1084 & C-O-C in glycidyl groups \\
1146 and 1234 & C-F in perfluoroalkyl \\
1685 & C=O in the acrylic segment \\
\hline
\end{tabular}




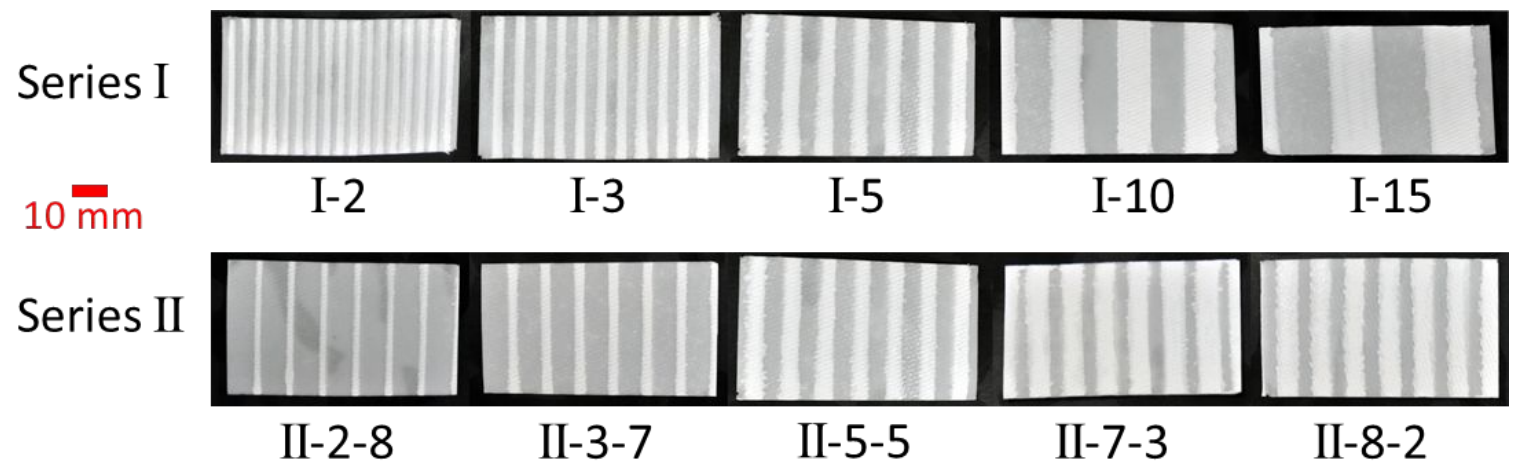

Figure S3. Digital photos of coated glass fiber filter with different patterns. 


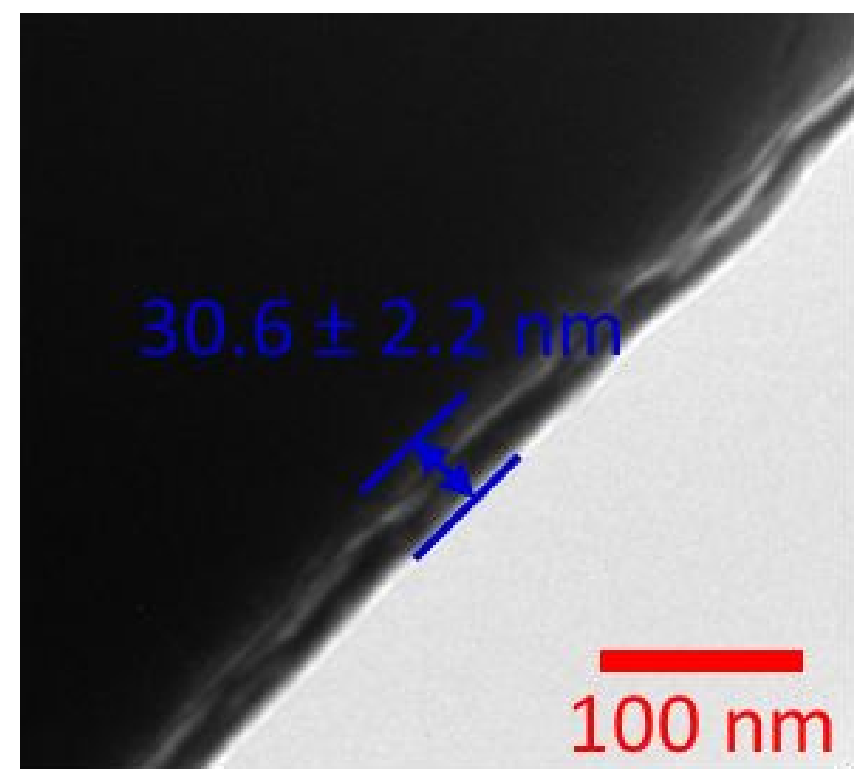

Figure S4. TEM image of a spray-coated single fiber.

Figure S4 shows a typical TEM image of the coated fiber. The dark region shows the crosssection of the cut fiber. The coating thickness of the PFAP/FAS layer is $30.6 \pm 2.2 \mu \mathrm{m}$ (measured from TEM images). The thickness value was an average of 30 measurements. 

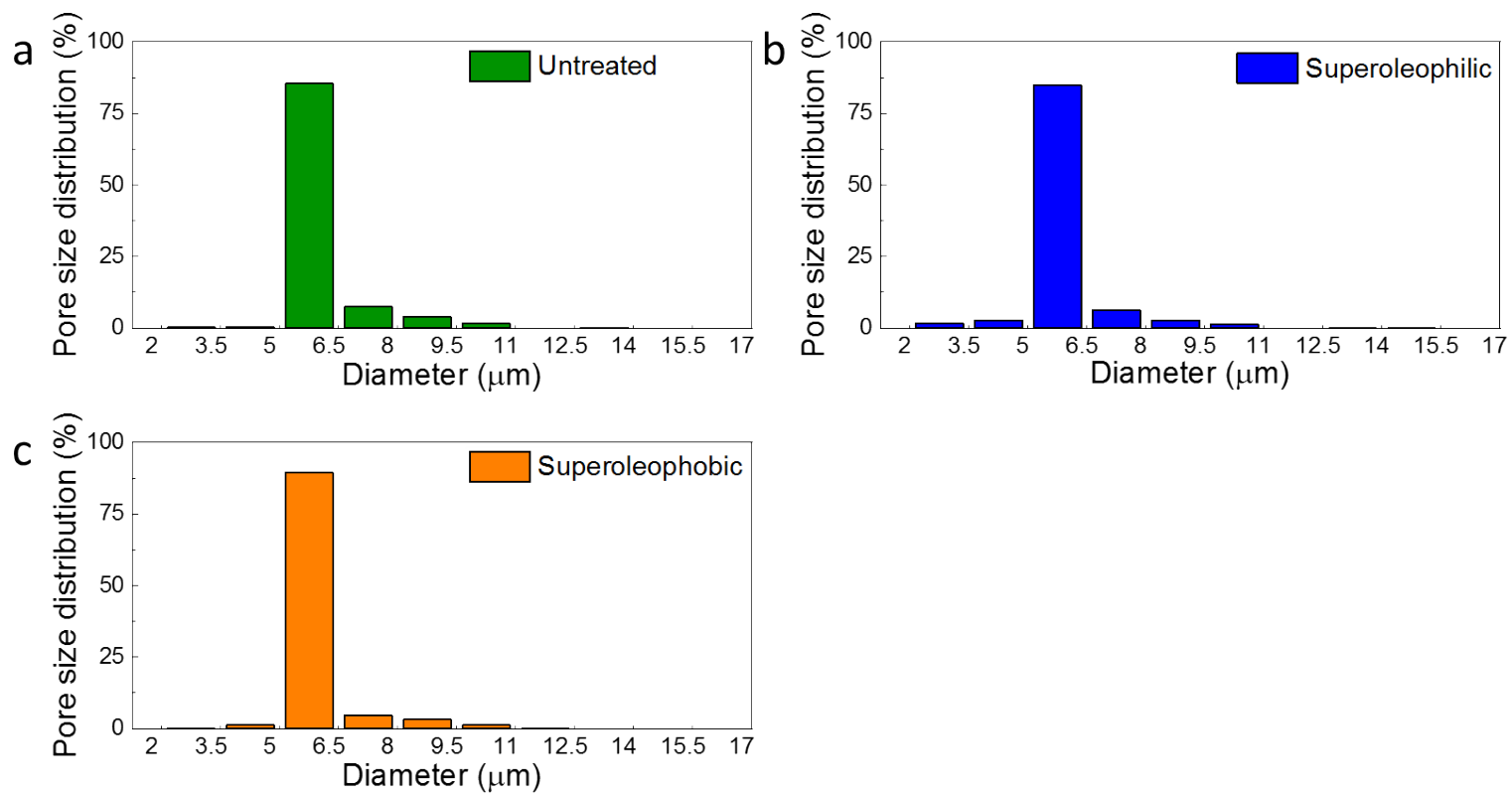

Figure S5. Pore size distribution (a) untreated sample, (b) dip-coated superoleophilic sample, and (c) spray-coated superoleophobic sample.

The mean pore size of the untreated filter was $6.68 \mu \mathrm{m}$, of superoleophilic filter was $6.61 \mu \mathrm{m}$, and that of the superoleophobic filter was $6.62 \mu \mathrm{m}$. The three samples showed a similar pore size distribution profile. It indicates that the coating treatments had little effect on average pore size and pore size distribution. 

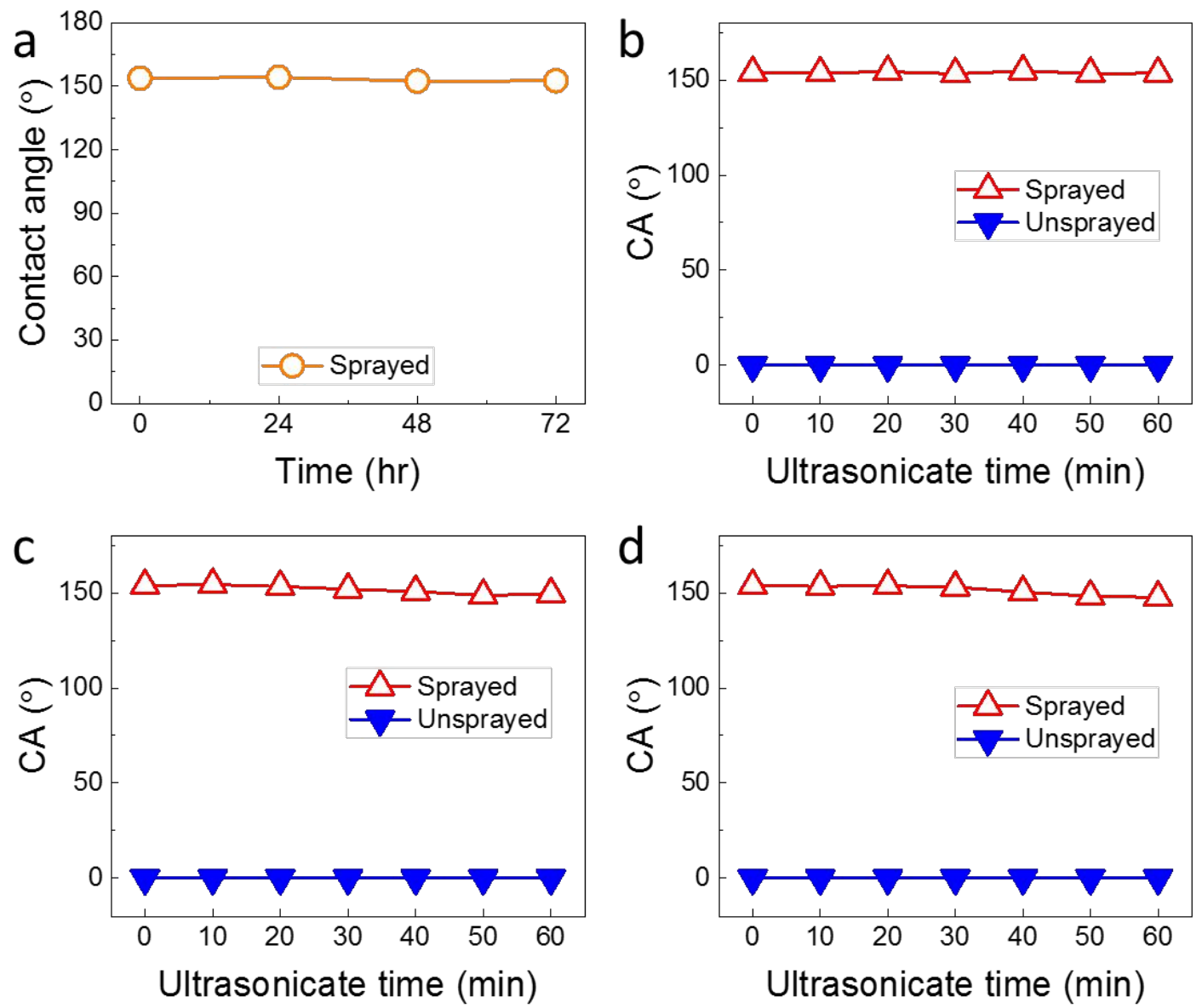

Figure S6. The durability of the filter with in-plane patterns with extreme wettability contrast. (a) Oil contact angle of filters after thermal durability test, heated at $150^{\circ} \mathrm{C}$ for three days. (b-d) The oil contact angle of filters after ultrasonication in (b) water, (c) ethanol, and (d) DEHS.

In brief, the treated samples can withstand heating at $150{ }^{\circ} \mathrm{C}$ for 72 hours without changing surface properties (Figure S6a). After ultrasonication in water, ethanol or DEHS for 1 hour, the surface features did not change as well. 


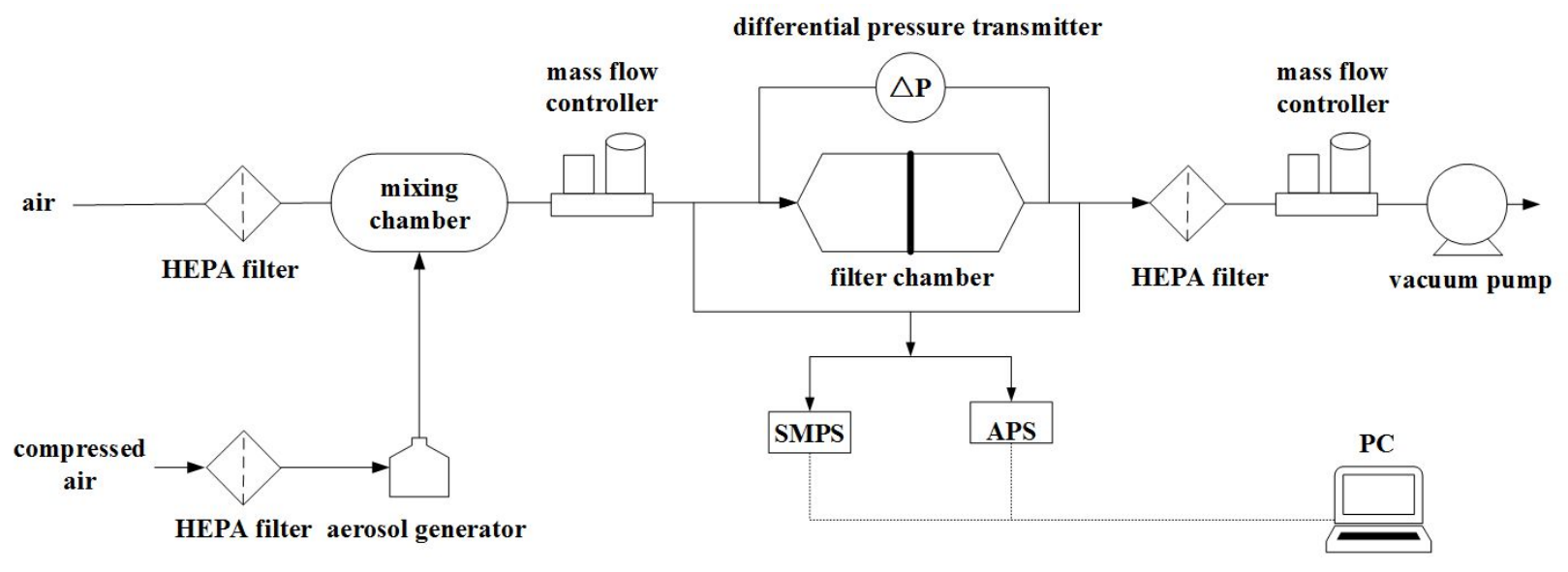

Figure S7. Schematic apparatus for filtration performance test 


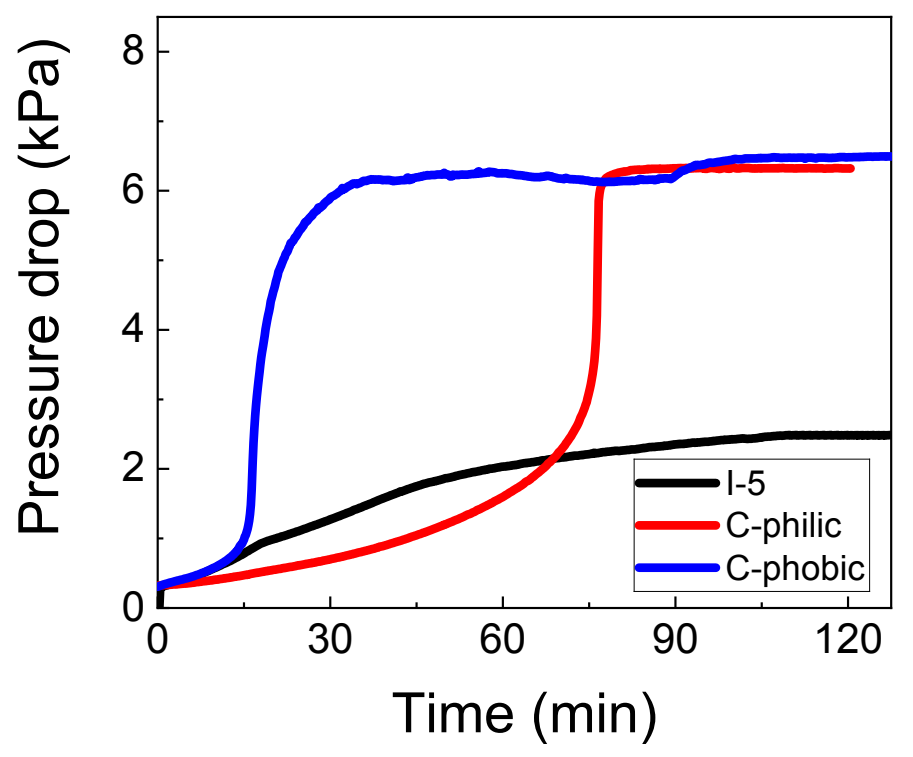

Figure S8. Single-layer pressure drop profile of superoleophobic filter, superoleophilic filter, and I-5 pattered filter.

Figure S8 shows the pressure drop profile of single-layer filter change with time. A pseudosteady state reaches when the pressure drop remains unchanged. The filtration tests for singlelayer filters last over 120 mins, while those of dual-layer filters over 150 mins. The filtration processes for different samples are ongoing with the same time and same testing condition (oil mist inlet concentration and airflow rate) to ensure the comparability of the experimental results. 

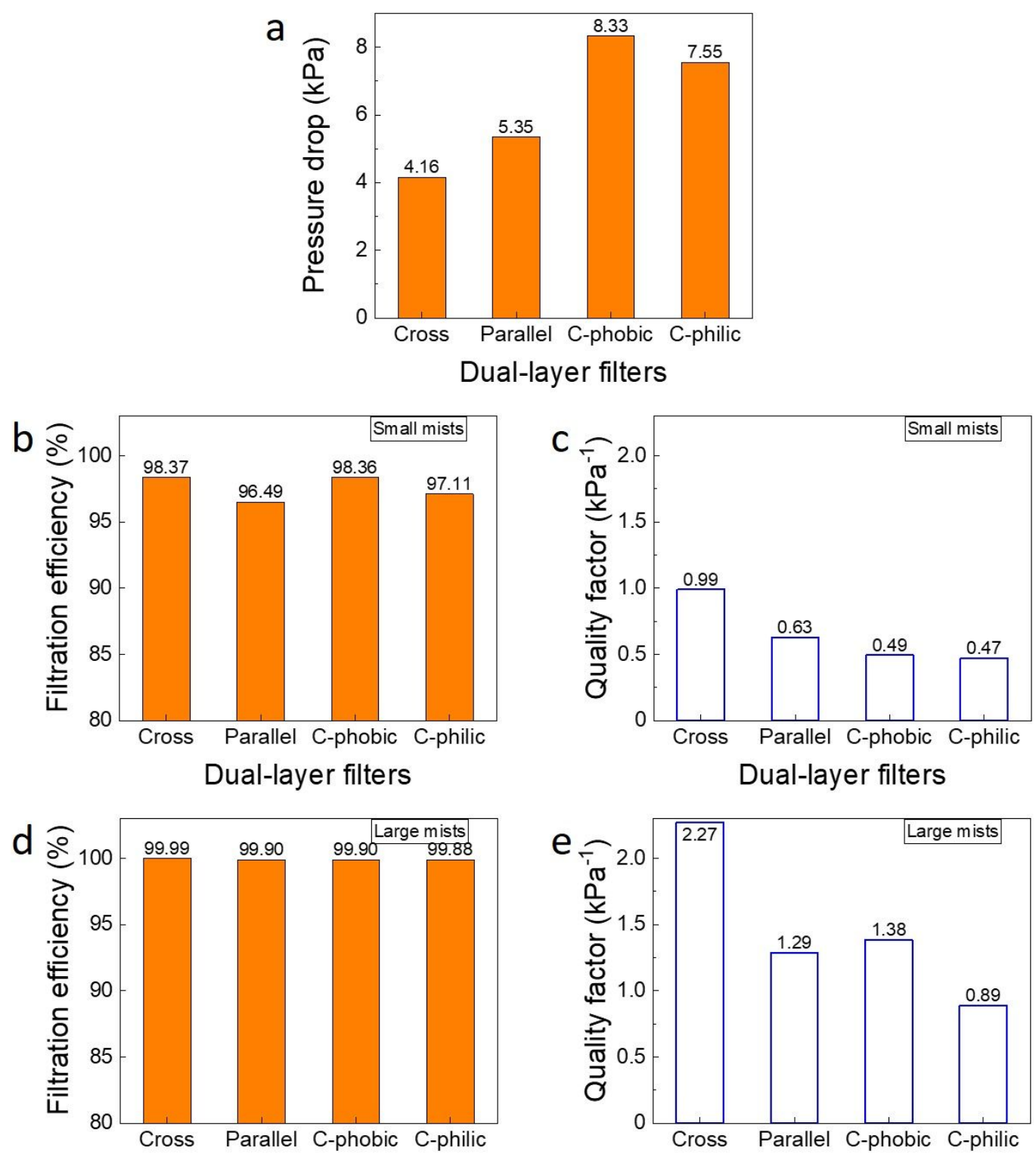

Figure S9. Filtration performance of dual-layer I-5 (or //-5-5) filters in different stacking arrangements and comparison with control filters: (a) pressure drop; (b) \& (c) filtration efficiency and quality factor for small oil mists; (d) \& (e) filtration efficiency and quality factor for large oil mists. 

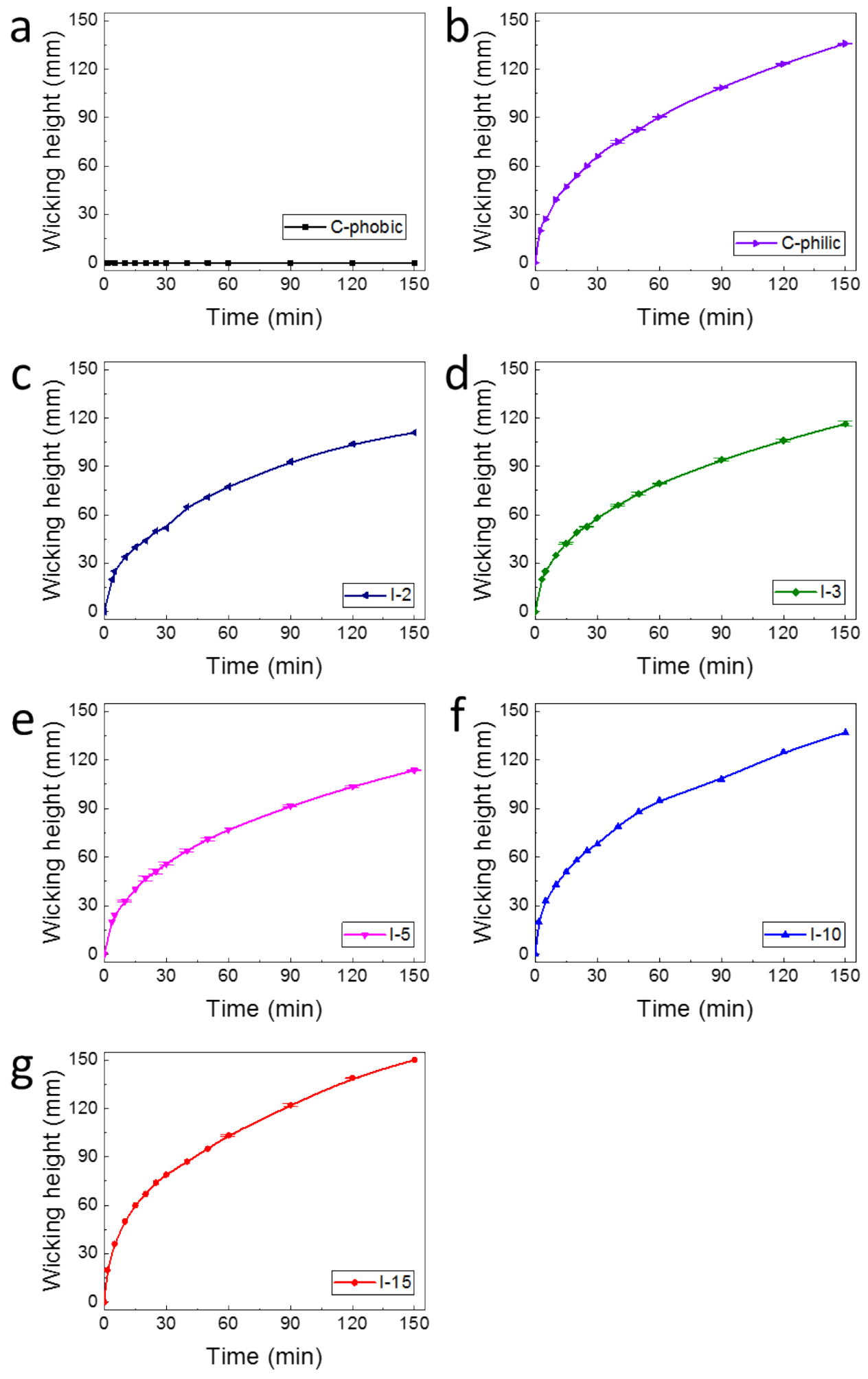
Figure S10. DEHS Wicking height ( $/$ ) changes with time ( $t)$. (a-b) Control samples: C-phobic and C-philic; (c-g) the series / samples, I-2, I-3, I-5, I-10, and I-15.
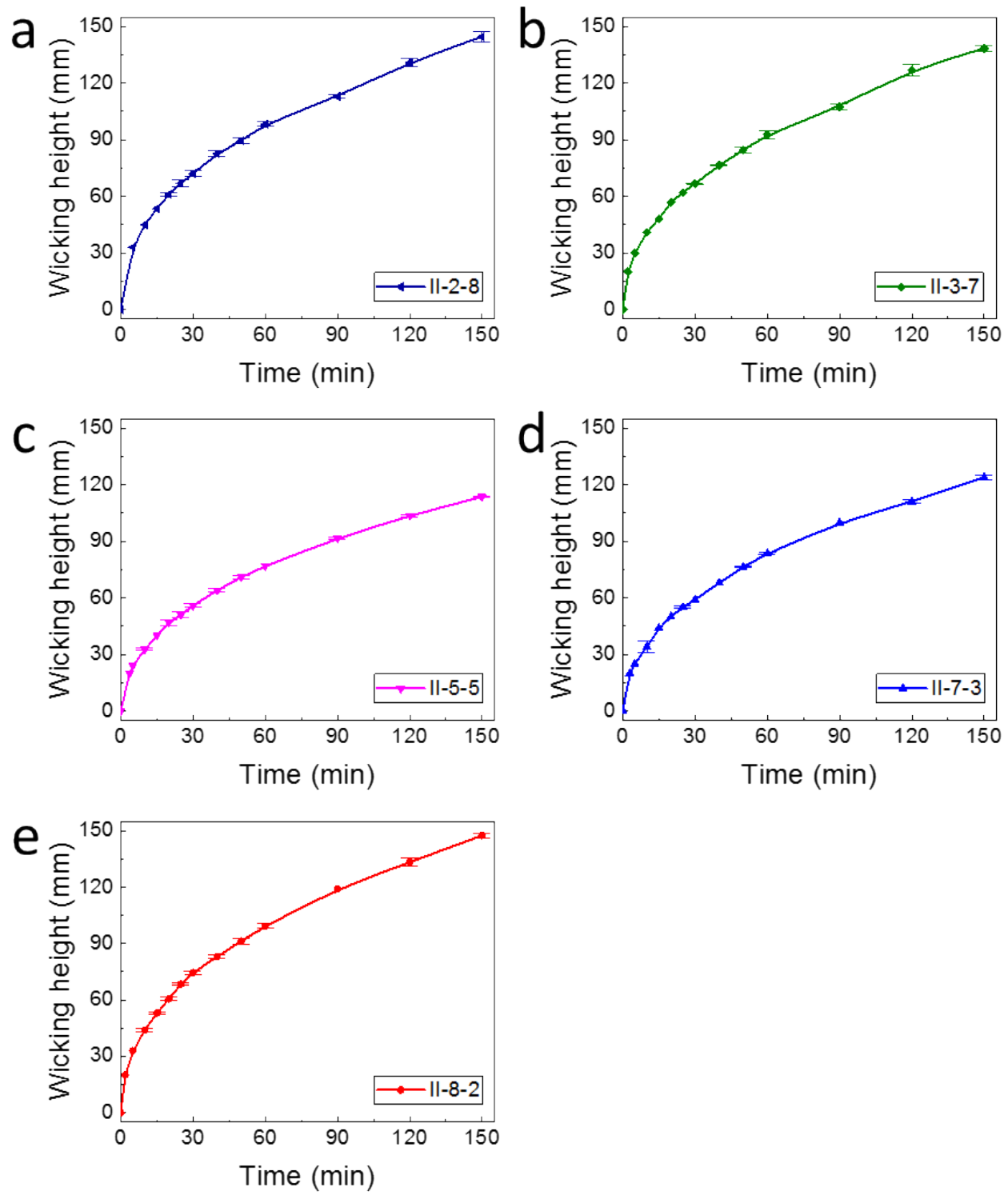

Figure S11. DEHS Wicking height changes with time. (a-e) The series //samples, /I-2-8, I/-3-7, /I-5-5, /I-7-3, and //-8-2. 

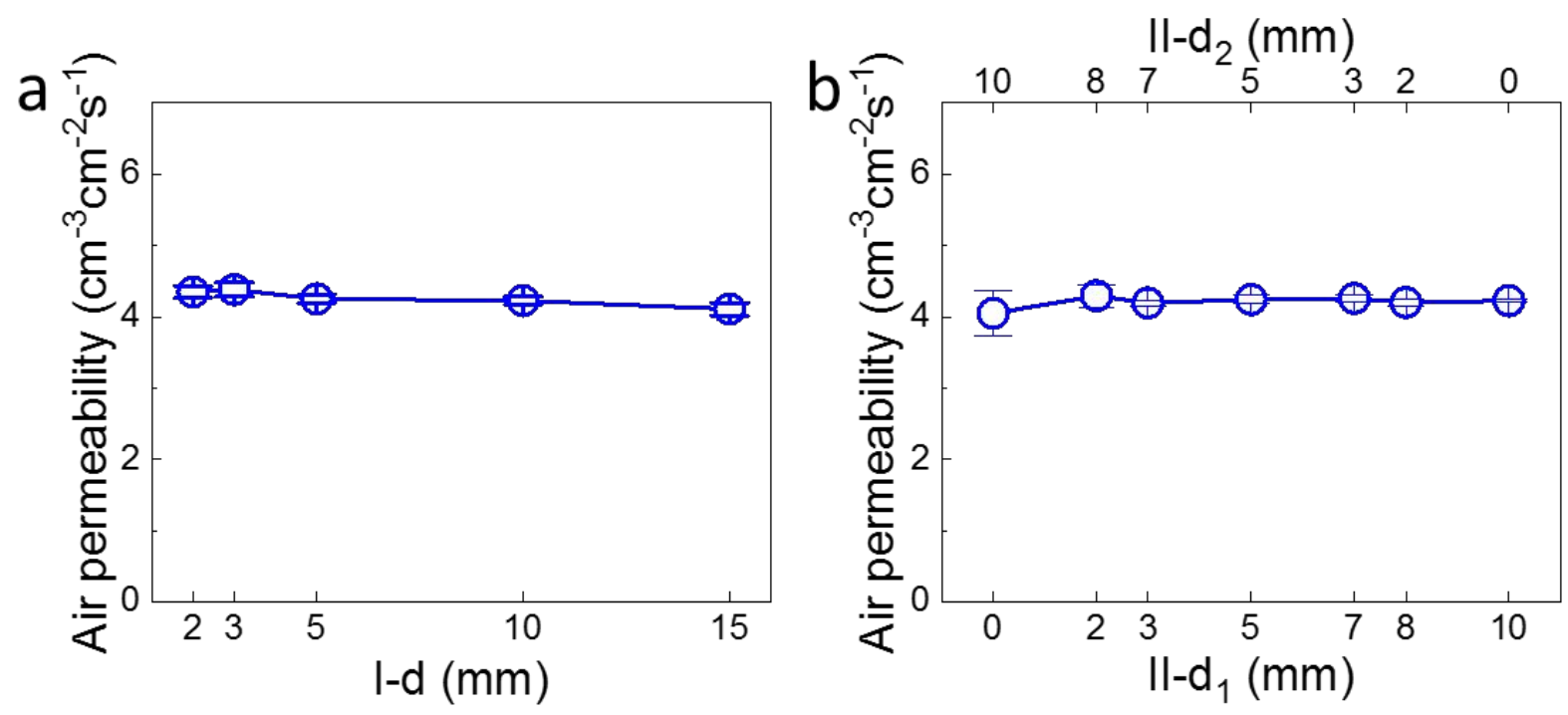

Figure S12. Air permeability (BS 5'636 Standard, test area of $5 \mathrm{~cm}^{2}$, test pressure of $96 \mathrm{~Pa}$ of the filter with in-plane patterns (a) Series / (b) Series II. 

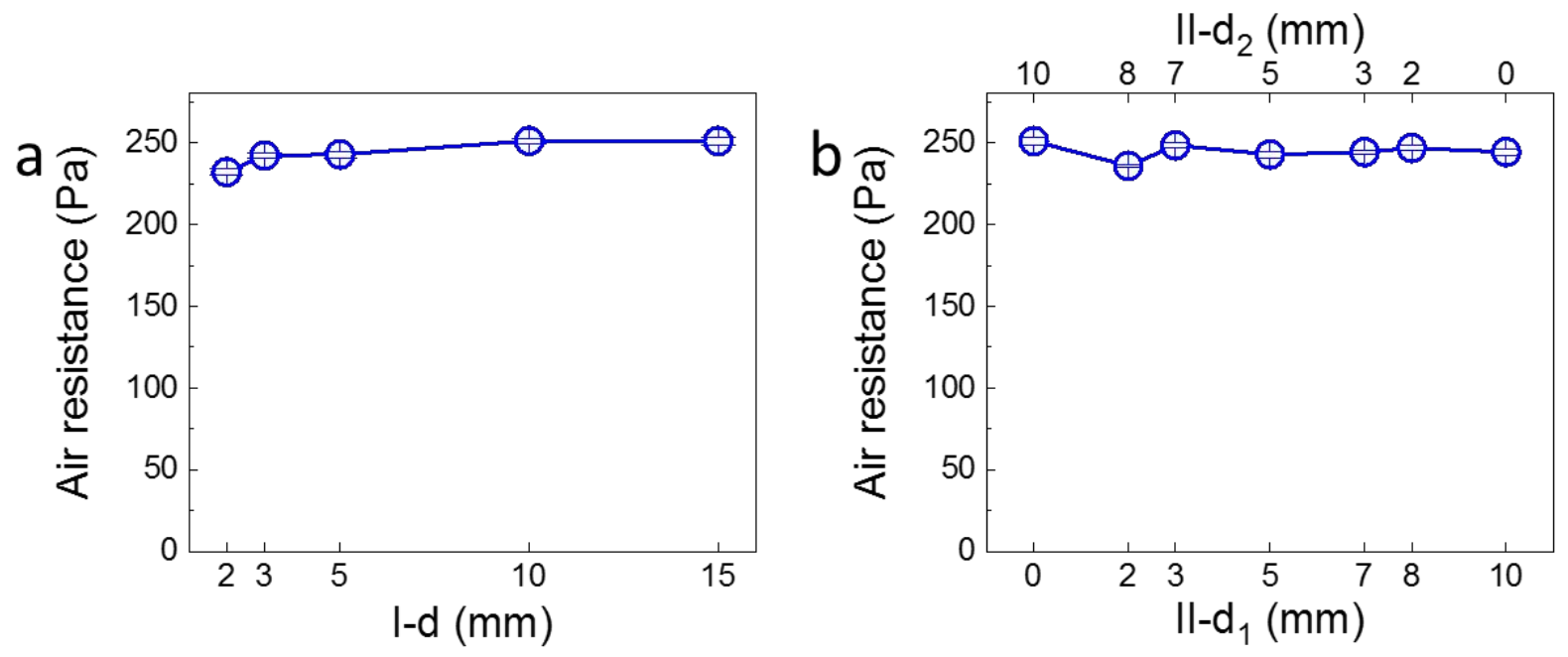

Figure S13. Dry state air resistance of patterned samples in different superoleophobic/ superoleophilic patterns: (a) Series I, (b) Series II.

The air resistance, also referred to as dry state pressure drop, was tested using the same face velocity with the oil mist filtration test. As shown in Figure S12, filters with different pattern widths and ratios have almost the same air resistance. Therefore, the influence of patterns on dry state pressure drop is minimal. 

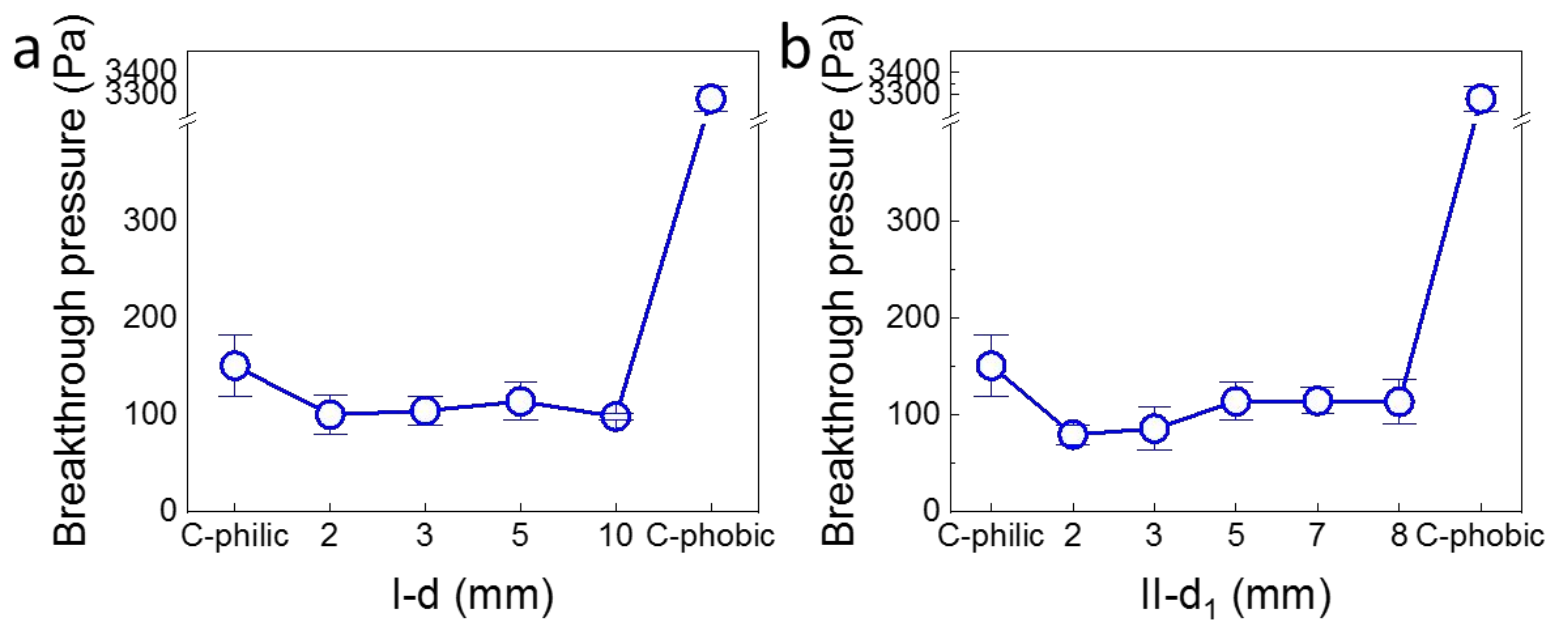

Figure S14. Breakthrough pressure of glass fiber samples in different in-plane superoleophobic /superoleophilic strip patterns (a) series I, and (b) series I/.

All the patterned samples had similar breakthrough pressures around $100 \mathrm{~Pa}$, which was much lower than the one with homogeneous superoleophobicity (3279.8 Pa) or superoleophilicity (150.8 Pa). The reason might be the superoleophilic stripe between two superoleophobic stripes could generate a capillary force to push the oil flow into the fibrous structure. In contrast, the superoleophobic samples only generated zero capillary force and repelled the oil to penetrate across the sample. 
Table S1. Spray parameters for preparing patterned filters

Series / samples

\begin{tabular}{|c|c|c|c|c|c|c|}
\hline \multirow{2}{*}{$\begin{array}{l}\text { Serie } \\
\text { sI }\end{array}$} & \multicolumn{2}{|c|}{ Prepared filter sample } & \multicolumn{2}{|c|}{ Plastic mask } & \multirow[b]{2}{*}{$\begin{array}{l}\text { Spra } \\
\text { y } \\
\text { cycle } \\
\text { s on } \\
\text { both } \\
\text { sides }\end{array}$} & \multirow[b]{2}{*}{$\begin{array}{l}\text { Superoleophob } \\
\text { ic proportion }\end{array}$} \\
\hline & $\begin{array}{l}\text { Superoleopho } \\
\text { bic width (mm) }\end{array}$ & $\begin{array}{l}\text { Superoleophil } \\
\text { ic width (mm) }\end{array}$ & $\begin{array}{l}\text { Hollo } \\
\text { w } \\
\text { width } \\
(\mathrm{mm})\end{array}$ & $\begin{array}{l}\text { Mas } \\
\mathrm{k} \\
\text { widt } \\
\mathrm{h} \\
(\mathrm{mm} \\
)\end{array}$ & & \\
\hline I-15 & 15 & 15 & 13.5 & 16.5 & 30 & $50 \%$ \\
\hline I-10 & 10 & 10 & 8.5 & 11.5 & 20 & $50 \%$ \\
\hline $1-5$ & 5 & 5 & 3.5 & 6.5 & 13 & $50 \%$ \\
\hline I-3 & 3 & 3 & 2 & 4 & 10 & $50 \%$ \\
\hline I-2 & 2 & 2 & 1 & 3 & 8 & $50 \%$ \\
\hline
\end{tabular}

Series // samples

\begin{tabular}{|c|c|c|c|c|c|c|}
\hline \multirow{2}{*}{$\begin{array}{l}\text { Serie } \\
\text { s II }\end{array}$} & \multicolumn{2}{|c|}{ Prepared filter sample } & \multicolumn{2}{|c|}{ Plastic mask } & \multirow[b]{2}{*}{$\begin{array}{l}\text { Spra } \\
\text { y } \\
\text { cycle } \\
\text { s on } \\
\text { both } \\
\text { sides }\end{array}$} & \multirow[b]{2}{*}{$\begin{array}{l}\text { Superoleophob } \\
\text { ic proportion }\end{array}$} \\
\hline & $\begin{array}{l}\text { Superoleopho } \\
\text { bic width (mm) }\end{array}$ & $\begin{array}{l}\text { Superoleophil } \\
\text { ic width (mm) }\end{array}$ & $\begin{array}{l}\text { Hollc } \\
\text { w } \\
\text { widtt } \\
\text { (mm }\end{array}$ & $\begin{array}{l}\text { Mas } \\
\mathrm{k} \\
\text { widt } \\
\mathrm{h} \\
(\mathrm{mm} \\
)^{2}\end{array}$ & & \\
\hline II-8-2 & 8 & 2 & 6.5 & 3.5 & 14 & $80 \%$ \\
\hline |I-7-3 & 7 & 3 & 5 & 5 & 13 & $70 \%$ \\
\hline II-5-5 & 5 & 5 & 3.5 & 6.5 & 13 & $50 \%$ \\
\hline II-3-7 & 3 & 7 & 2 & 8 & 10 & $30 \%$ \\
\hline||$-2-8$ & 2 & 8 & 1 & 9 & 8 & $20 \%$ \\
\hline
\end{tabular}


Table S2. Oil-mist filtration performance of single-layer patterned filters

\begin{tabular}{|c|c|c|c|c|c|c|}
\hline & \multirow{2}{*}{$\begin{array}{l}\text { Superoleophobic } \\
\text { / superoleophilic } \\
\text { width }(\mathrm{mm} / \mathrm{mm})\end{array}$} & \multirow{2}{*}{$\begin{array}{l}\text { Pressure } \\
\text { drop } \\
(\mathrm{kPa})\end{array}$} & \multicolumn{2}{|c|}{ Small mists } & \multicolumn{2}{|c|}{ Large mists } \\
\hline & & & $\begin{array}{l}\text { Efficiency } \\
(\%)\end{array}$ & $\begin{array}{l}\text { Quality factor } \\
\left(\mathrm{kPa}^{-1}\right)\end{array}$ & $\begin{array}{l}\text { Efficiency } \\
(\%)\end{array}$ & $\begin{array}{l}\text { Quality } \\
\text { factor } \\
\left(\mathrm{kPa}^{-1}\right)\end{array}$ \\
\hline \multirow{5}{*}{$\begin{array}{l}\frac{5}{5} \\
\frac{0}{3} \\
\frac{1}{c} \\
\frac{1}{0} \\
\frac{0}{0} \\
\frac{.0}{0}\end{array}$} & |-15 & 4.77 & 89.49 & 0.47 & 98.85 & 0.94 \\
\hline & $\mid-10$ & 3.82 & 93.54 & 0.72 & 99.69 & 1.56 \\
\hline & $1-5$ & 2.49 & 93.23 & 1.08 & 99.56 & 2.18 \\
\hline & I-3 & 4.92 & 89.49 & 0.46 & 99.14 & 0.97 \\
\hline & I-2 & 6.07 & 86.49 & 0.33 & 99.24 & 0.80 \\
\hline \multirow{5}{*}{ 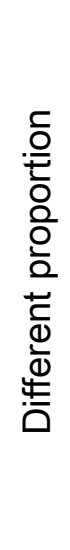 } & |I-8-2 & 5.06 & 88.05 & 0.42 & 98.94 & 0.90 \\
\hline & II-7-3 & 3.45 & 89.57 & 0.66 & 98.63 & 1.24 \\
\hline & II-5-5 & 2.49 & 93.23 & 1.08 & 99.56 & 2.18 \\
\hline & II-3-7 & 6.03 & 89.90 & 0.38 & 98.86 & 0.74 \\
\hline & |I-2-8 & 5.49 & 87.72 & 0.38 & 98.59 & 0.78 \\
\hline
\end{tabular}


Table S3. Oil-mist filtration performance of dual-layer patterned filters

\begin{tabular}{|c|c|c|c|c|c|c|}
\hline & \multirow{2}{*}{$\begin{array}{l}\text { Superoleophobic/ } \\
\text { superoleophilic } \\
\text { width }(\mathrm{mm} / \mathrm{mm})\end{array}$} & \multirow{2}{*}{$\begin{array}{l}\text { Pressure } \\
\text { drop } \\
(\mathrm{kPa})\end{array}$} & \multicolumn{2}{|l|}{ Small mists } & \multicolumn{2}{|c|}{ Large mists } \\
\hline & & & $\begin{array}{l}\text { Efficiency } \\
(\%)\end{array}$ & $\begin{array}{l}\text { Quality factor } \\
\left(\mathrm{kPa}^{-1}\right)\end{array}$ & $\begin{array}{l}\text { Efficiency } \\
(\%)\end{array}$ & $\begin{array}{l}\text { Quality } \\
\text { factor } \\
\left(\mathrm{kPa}^{-1}\right)\end{array}$ \\
\hline \multirow{5}{*}{ 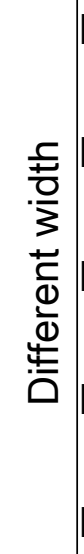 } & $\mid-15$ & 6.45 & 97.23 & 0.56 & 99.90 & 1.06 \\
\hline & |-10 & 6.57 & 98.77 & 0.67 & 99.90 & 1.04 \\
\hline & $1-5$ & 4.16 & 98.37 & 0.99 & 99.99 & 2.27 \\
\hline & I-3 & 6.51 & 98.43 & 0.64 & 99.84 & 0.98 \\
\hline & $\mid-2$ & 8.07 & 97.37 & 0.45 & 99.79 & 0.77 \\
\hline \multirow{5}{*}{$\begin{array}{l}\frac{c}{0} \\
\frac{0}{0} \\
\frac{0}{0} \\
\frac{0}{2} \\
\frac{c}{0} \\
\frac{0}{0} \\
\frac{4}{0}\end{array}$} & |II-8-2 & 5.56 & 98.44 & 0.75 & 99.87 & 1.19 \\
\hline & |II-7-3 & 4.48 & 97.77 & 0.85 & 99.83 & 1.43 \\
\hline & $\mid I-5-5$ & 4.16 & 98.37 & 0.99 & 99.99 & 2.27 \\
\hline & II-3-7 & 6.41 & 97.12 & 0.55 & 99.78 & 0.96 \\
\hline & ||I-2-8 & 6.75 & 97.15 & 0.53 & 99.85 & 0.96 \\
\hline
\end{tabular}


Table S4. Comparison of oil-mist filtration performance between different dual-layer filters

\begin{tabular}{|c|c|c|c|c|c|c|}
\hline \multirow[b]{2}{*}{ Sample } & \multirow{2}{*}{$\begin{array}{l}\text { Pressur } \\
\text { e drop } \\
(\mathrm{kPa})\end{array}$} & \multicolumn{2}{|c|}{ Small mists } & \multicolumn{2}{|c|}{ Large mists } & \multirow[b]{2}{*}{$\begin{array}{l}\text { Compare with the } \\
\text { untreated sample }\end{array}$} \\
\hline & & $\begin{array}{l}\text { Efficiency } \\
(\%)\end{array}$ & $\begin{array}{l}\text { Quality } \\
\text { factor } \\
\left(\mathrm{kPa}^{-1}\right)\end{array}$ & $\begin{array}{l}\text { Efficiency } \\
(\%)\end{array}$ & $\begin{array}{l}\text { Quality } \\
\text { factor } \\
\left(\mathrm{kPa}^{-1}\right)\end{array}$ & \\
\hline Untreated & 7.60 & 96.29 & 0.43 & 99.43 & 0.68 & $\begin{array}{l}\text { N/A } \\
\Delta \mathrm{P} \uparrow 9.6 \% \\
\text { Small: } \varepsilon \uparrow 2.1 \% \text {, }\end{array}$ \\
\hline C-phobic & 8.33 & 98.36 & 0.49 & 99.90 & 1.38 & $\begin{array}{l}\mathrm{Q}_{\mathrm{F}} \uparrow 14.0 \% \\
\text { Large: } \varepsilon \uparrow 0.5 \%, \\
\mathrm{Q}_{\mathrm{F}} \uparrow 102.9 \% \\
\Delta \mathrm{P} \downarrow 0.7 \%,\end{array}$ \\
\hline C-philic & 7.55 & 97.11 & 0.47 & 99.88 & 0.89 & $\begin{array}{l}\text { Small: } \varepsilon \uparrow \quad 0.9 \% \text {, } \\
Q_{F} \uparrow 9.3 \%\end{array}$ \\
\hline & & & & & & $\begin{array}{l}\text { Large: } \varepsilon \uparrow 0.5 \%, \\
\mathrm{Q}_{\mathrm{F}} \uparrow 30.9 \% \\
\Delta \mathrm{P} \uparrow 9.6 \%,\end{array}$ \\
\hline Superoleophobic & 8.33 & 99.44 & 0.62 & 99.99 & 2.12 & $\begin{array}{l}\text { Small: } \varepsilon \uparrow ~ 3.3 \%, \\
Q_{F} \uparrow 44.2 \%\end{array}$ \\
\hline 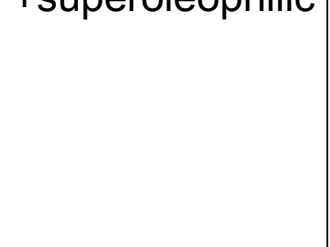 & & & & & & $\begin{array}{l}\text { Large: } \varepsilon \uparrow 0.6 \%, \\
Q_{F} \uparrow 211.8 \% \\
\Delta P \uparrow 22.2 \%,\end{array}$ \\
\hline $\begin{array}{l}\text { Directional oil } \\
\text { transport+ }\end{array}$ & 9.29 & 99.45 & 0.56 & 99.99 & 1.75 & $\begin{array}{l}\text { Small: } \varepsilon \uparrow ~ 3.3 \% \text {, } \\
Q_{F} \uparrow 30.2 \%\end{array}$ \\
\hline superoleophobic & & & & & & $\begin{array}{l}\text { Large: } \varepsilon \uparrow 0.6 \% \text {, } \\
Q_{F} \uparrow 157.4 \%\end{array}$ \\
\hline
\end{tabular}




\begin{tabular}{|c|c|c|c|c|c|c|}
\hline \multirow{5}{*}{ This work } & \multirow{5}{*}{4.16} & \multirow{5}{*}{98.37} & \multirow{5}{*}{0.99} & \multirow{5}{*}{99.99} & \multirow{5}{*}{2.27} & $\Delta \mathrm{P} \downarrow 45.3 \%$ \\
\hline & & & & & & Small: $\varepsilon \uparrow 2.2 \%$, \\
\hline & & & & & & $Q_{F} \uparrow 130.2 \%$ \\
\hline & & & & & & Large: $\varepsilon \uparrow ~ 0.6 \%$, \\
\hline & & & & & & $Q_{F} \uparrow 233.8 \%$ \\
\hline
\end{tabular}

